\title{
Descending necrotizing mediastinitis after upper gastrointestinal endoscopy
}

An 83-year-old man presented with a 2week history of intermittent left-sided chest pain. His chest radiograph and electrocardiogram were unremarkable, and so upper gastrointestinal endoscopy was performed for screening purposes. The intubation and procedure were carried out without difficulty, although the patient gagged occasionally. There was no obvious evidence of a site of perforation. Shortly after endoscopy, the patient became distressed, complaining of diffuse cervical swelling and a severe sore throat. Examination at that time revealed subcutaneous emphysema of the neck. He had a fever, dyspnea, and hypoxemia. His laboratory results revealed a white blood cell count of $18000 / \mathrm{mm}^{3}$ and a C-reactive protein level of $22.5 \mathrm{mg} / \mathrm{dl}$. A chest radiograph showed pneumomediastinum and subcutaneous emphysema. Cervical and thoracic computed tomographic scans revealed cervical necrotizing fasciitis and descending necrotizing mediastinitis (Figure 1).

Endotracheal intubation was performed and he was started on broad-spectrum antibiotics. A thoracostomy tube was inserted; and a percutaneous catheter was inserted, from the cervical space along the route of infection. Bacteriological investigation did not reveal any microorganism. He underwent a gastrointestinal radiographic contrast study, but no evidence of esophageal perforation was found. The patient was treated conservatively with antibiotic therapy and insertion of a drainage catheter. He improved gradually, and was discharged 60 days after the upper gastrointestinal endoscopy.

Mediastinal and subcutaneous emphysema is a rare complication of routine gastrointestinal endoscopy. Several reports have described pneumomediastinum alone [1], retroperitoneal emphysema alone [2], or retroperitoneal, mediastinal, and subcutaneous emphysema with no demonstrable perforation $[3,4]$. These conditions were benign and self-limiting.

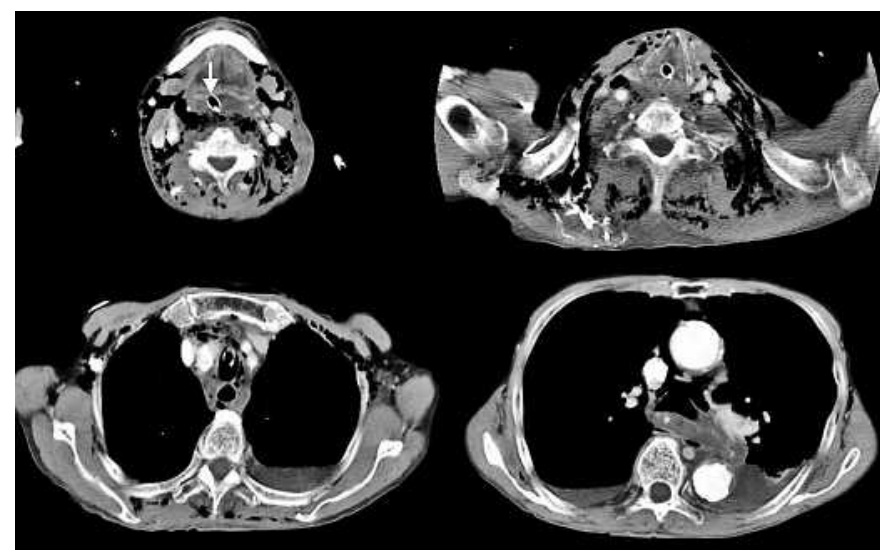

Figure 1 Cervicothoracic computed tomographic scans showing an abscess and a collection of gas in the cervical region and mediastinal space, subcutaneous emphysema, and bilateral pleural effusions. An endotracheal tube was inserted (arrow).

In our case, the patient presented with severe sore throat shortly after endoscopy, so we considered that the air could initially have been forced through weak points in the oropharyngeal mucosa, the infection then spreading along the oropharyngeal space to reach the mediastinum. Descending necrotizing mediastinitis is an uncommon condition and is associated with a high mortality rate [5]. It is important that physicans should be alert to the fact that descending necrotizing mediastinitis can be a complication of routine upper gastrointestinal endoscopy.

\section{Endoscopy_UCTN_Code_CPL_1AH_2AB}

\section{K. Kaira, H. Yasuoka, T. Ichikawa,}

S. Oh-i, T. Hisada, T. Ishizuka, M. Mori

Department of Medicine and Molecular Science, Gunma University Graduate School of Medicine, Showa-machi, Maebashi, Gunma, Japan.

\section{References}

${ }^{1}$ Katz D, Cano R, Antonello M. Benign air dissection of the esophagus and stomach at fiberoptic endoscopy. Gastrointest Endosc 1972; 19: 72 -

${ }^{2}$ Martin WC, Nagai N, Diuer W et al. Benign retroperitoneal emphysema associated with upper gastrointestinal endoscopy. Arch Intern Med 1978; 138: 759-768

${ }^{3}$ Girardi A, Piazza I, Giunta G et al. Retroperitoneal, mediastinal and subcutaneous emphysema as a complication of routine upper gastrointestinal endoscopy. Endoscopy 1990; 22: 83-84

${ }^{4}$ Fireman Z, Bar-Ziv J, Lurie B. Retroperitoneal, mediastinal and subcutaneous emphysema as a complication of routine upper gastrointestinal endoscopy. Endoscopy 1994; 26: $329-330$

${ }^{5}$ Nakamori Y, Fujimi S, Ogura H et al. Conventional open surgery versus percutaneous catheter drainage in the treatment of cervical necrotizing fasciitis and descending necrotizing mediastinitis. AJR Am J Roentgenol 2004; 182: $1443-1449$

\section{Corresponding author}

\section{K. Kaira, M. D.}

Department of Medicine and

Molecular Science

Gunma University Graduate

School of Medicine

Showa-machi

Maebashi

Gunma 371-8511

Japan

Fax: $\quad+81-27-220-8130$

Email: kkaira1970@yahoo.co.jp 